УДК 621.626 .8

$10.17213 / 2075-2067-2020-4-91-97$

\title{
ПОВЫШЕНИЕ ЭКОНОМИЧЕСКОЙ ЭФФЕКТИВНОСТИ ОЧИСТКИ МЕЛИОРАТИВНЫХ КАНАЛОВ ЗА СЧЕТ ИЗМЕНЕНИЯ ОСНОВНЫХ ПАРАМЕТРОВ РЕЖУЩЕГО АППАРАТА КОСИЛКИ
}

\author{
(C) 2020 г. В. В. Грищенко
}

Новочеркасский инженерно-мелиоративный институт имени А. К. Кортунова (филиал), Донской государственный аграрный университет, 2. Новочеркасск, Россия

Целью исследования является повышение экономической эффективности режущего аппарата косилки за счет определения оптимальных параметров ножа и обеспечения долговечности машины. При решении данной задачи учитывались скорость резания, масса ножа и его геометрические параметры, необходимые для срезания сорной растительности и удаления срезанной массы из зоны резания.

Методологическую базу исследования представляют основы теории бесподпорного резания В.П. Горячкина, а также анализ теоретических исследований сопротивляемости стебля отгибу, проведенных В. Ф. Семеновым и Е. С. Босым.

Результаты исследования. Для обеспечения экономической эффективности были определены новые физические параметры иепи, что позволило сохранить работоспособность ротора в иелом. Старая иепь была заменена на новую, что повлекло за собой изменение геометрических параметров цуепного ножа. Экономическое обоснование позволило использовать новый цепной нож без изменения конструкции косилки КР-2,5K.

Перспективу исследования составляет дальнейший анализ прочности звена режущего аппарата мелиоративной косилки в различных условиях перегрузки.

Ключевые слова: косилка; ротор; режущий аппарат; сила резания; масса ножа; бесподпорное резание; нож; лезвие ножа.

\section{IMPROVING ECONOMIC EFFICIENCY CLEANING OF RECLAMATION CHANNELS BY CHANGING THE MAIN PARAMETERS OF THE MOWER CUTTING DEVICE}

\section{(C) 2020 V. V. Grishchenko}

Novocherkassk Reclamation Engineering Institute named after A. K. Kortunov (branch), Donskoy State Agrarian University, Novocherkassk, Russia

The purpose of the study is to increase the economic efficiency of the mower's cutting device by determining the optimal parameters of the knife and ensuring the machine's durability. When solving this problem, the cutting speed, weight of the knife and its geometric parameters necessary for cutting weeds and removing the cut mass from the cutting zone were taken into account.

The methodological base of the study is the basis of V.P. Goryachkin's theory of unsupported cutting, as well as the analysis of theoretical studies of stem bending resistance conducted by V.F. Semenov and E. S. Bosoy. 
The results of the study. In order to ensure economic efficiency, new physical parameters of the chain were determined, which allowed maintaining the efficiency of the rotor as a whole. The old chain was replaced with a new one, which led to a change in the geometric parameters of the chain knife. The economic justification allowed using a new chain knife without changing the design of the KR-2,5K mower.

The prospect of the study. Further analysis of the strength of the cutting unit of the reclamation mower under various overload conditions is a promising research project.

Key words: mower; rotor; cutting device; cutting force; weight of the knife; unsupported cutting; knife; blade of the knife.

Введение. Из всего комплекса мер по уходу за мелиоративными системами окашивание каналов и других ее элементов является наиболее трудоемкой технологической операцией. Эта операция должна проводиться от трех до четырех раз за сезон, в зависимости от погодных условий [5]. Использование современных средств механизации позволяет выполнять очистку мелиоративных каналов от сорной растительности оперативно в сжатые сроки, но режущие аппараты этих косилок приспособлены для работы в условиях только горизонтального среза.

Анализ показал, что мелиоративные косилки для окашивания откосов и берм каналов, как отечественные, так и зарубежные, оснащаются в основном двумя типами режущих аппаратов [6]: режущими аппаратами возвратно-поступательного действия и роторными с вертикальной осью вращения. В результате их применения на очищаемом участке канала остаются длинные срезанные стебли растений, которые необходимо удалить за пределы очищаемого участка. По существующей технологии для сгребания срезанной растительности на каналах применяют либо грабли, либо специальное сменное оборудование к косилкам. Однако существуют отечественные разработки, которые способны срезать растительность на склонах и выполнять несколько технологических операций за один проход. Среди них косилка со шнековым режущим аппаратом КОС-2,5 и роторная косилка с цепным ножом КР-2,5К, которые скашивают, измельчают и транспортируют измельченную массу из зоны резания. В нашем случае мы оптимизируем роторный режущий аппарат с вертикальной осью вращения косилки КР-2,5К.
Ведущие методологические подходы изучения процесса резания. В отличие от ножей, жёстко закреплённых на роторе, ножи косилки КР-2,5К имеют гибкую конструкцию. Они способны отклоняться при встрече с непреодолимым препятствием, что исключает поломку ножа и повышает надёжность режущего аппарата, но в этом случае возможно образование огрехов (непрокошенных участков). При применении устройств слежения, которые автоматически отводят ротор косилки от непреодолимого препятствия, огрехи остаются бо́льшими по протяженности, чем у цепного ножа. Сами устройства слежения требуют точной настройки и квалифицированного обслуживания, что удорожает конструкцию косилки.

Ранее нами было рассмотрено динамическое уравновешивание ротора [4], который набрал необходимую кинетическую энергию для срезания сорной растительности при раскручивании ротора до минимально возможной скорости вращения.

Дифференциальное уравнение вращения ножа относительно шарнира [1]:

$$
J \frac{d \omega_{1}}{d t}=\sum M
$$

где $J$ - момент инерции ножа относительно шарнира; $\omega_{1}$ - угловая скорость вращения ножа относительно шарнира; $\sum M$ - сумма моментов всех внешних сил, действующих на нож.

Сделан вывод, что ротор динамически будет уравновешен, если импульс реакции шарнира ножа равен нулю $S_{u}=0$, т.е.:

$$
\left[\frac{J}{b}-m \cdot c\right] \cdot \omega_{1}=0 \quad \text { или } \quad \frac{J}{b}-m \cdot c=0 .
$$




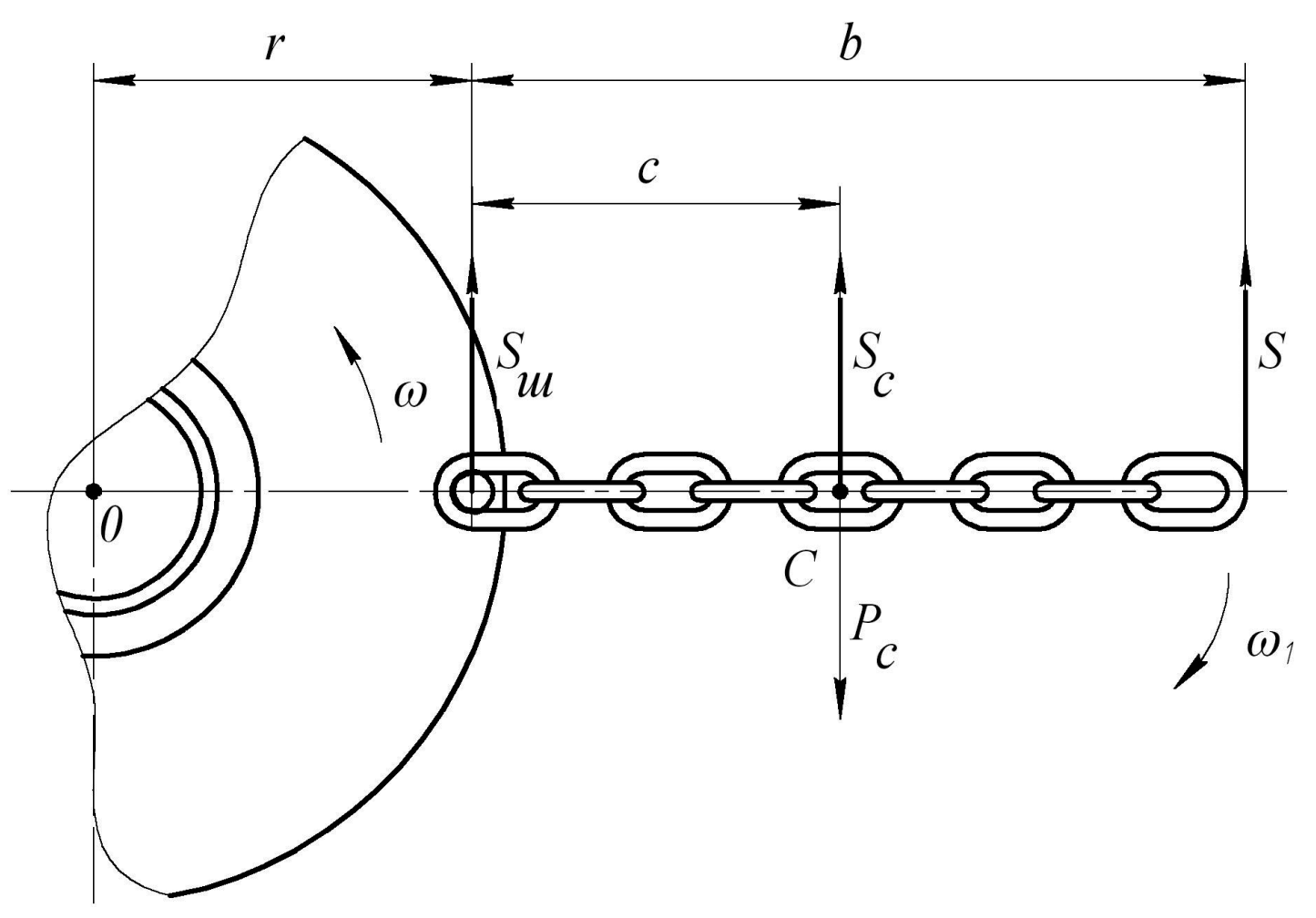

Рис. 1. Схема действия импульсов на нож в момент удара его по стеблю

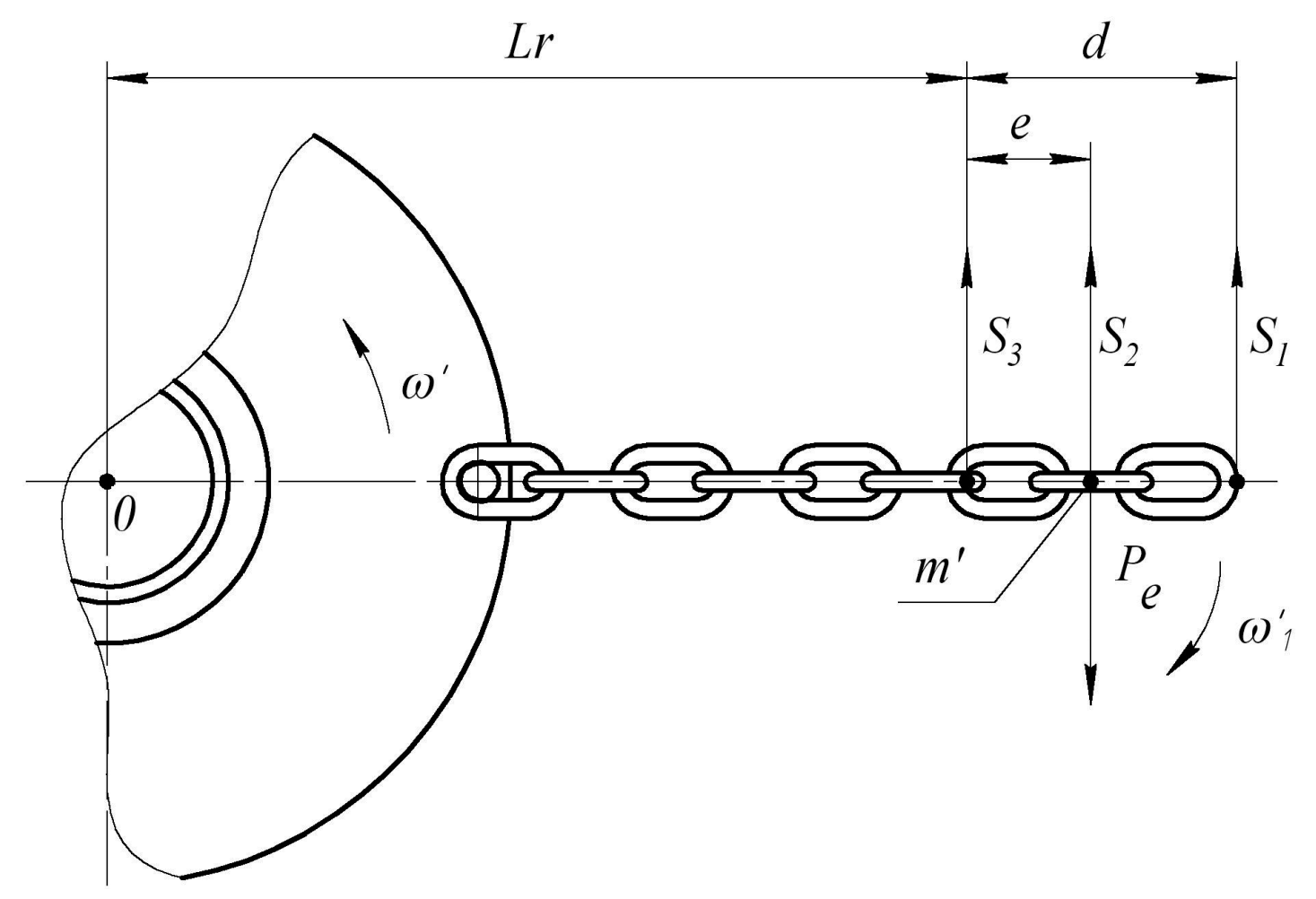

Рис. 2. Схема действия импульсов на крайние звенья ножа 
Так как

$$
J=m \cdot \rho^{2},
$$

где $\rho$ - радиус инерции ножа, то уравнение (2) примет вид:

$$
\frac{m \cdot \rho^{2}}{b}-m \cdot c=0
$$

откуда $\rho^{2}$ равно:

$$
\rho^{2}=b \cdot c .
$$

Таким образом ротор с ножами косилки будет уравновешен, если размеры и массу ножа выбрать так, чтобы квадрат радиуса инерции ножа $\rho$ равнялся произведению длины ножа на расстояние от центра тяжести до шарнира подвески.

Теперь рассмотрим только крайние три звена ножа (цепи), которые участвуют в процессе срезания сорной растительности и её транспортирования из зоны резания (рис. 2). Дифференциальное уравнение вращения ножа относительно новой точки шарнира (1) примет вид:

$$
J^{\prime} \frac{d \omega_{1}^{\prime}}{d t}=\sum M
$$

Если перейти к среднему ускорению, то получим:

$$
J^{\prime} \frac{\Delta \omega_{1}^{\prime}}{\Delta t}=M,
$$

где $\quad \Delta \omega_{1}^{\prime}=\omega_{1}^{\prime}-\omega_{0}^{\prime}$.

Так как $\omega_{1}^{\prime}=0$, имеем $\Delta \omega_{1}^{\prime}=\omega_{1}^{\prime}$ и формула (7) примет следующий вид:

$$
J \cdot \omega_{1}^{\prime}=M \cdot \Delta t .
$$

Момент $M$ есть произведение силы на плечо ее приложения, тогда:

$$
J \cdot \omega_{1}^{\prime}=P_{e} \cdot d \cdot \Delta t .
$$

Так как произведение $P_{e} \cdot \Delta t$ есть не что иное, как реакция $S_{1}$, то:

$$
J^{\prime} \cdot \omega_{1}^{\prime}=S_{1} \cdot d .
$$

Реакция в шарнире $S_{3}$ будет равна:

$$
S_{3}=S_{1}-S_{2} .
$$

Так как $S_{1}$ равно:

$$
S_{1}=\frac{J^{\prime} \cdot \omega_{1}^{\prime}}{d}
$$

а импульс силы, приведенный к центру тяжести ножа, равен количеству движения:

$$
S_{2}=m^{\prime} \cdot V_{m^{\prime}}=m^{\prime} \cdot \omega_{1}^{\prime} \cdot e .
$$

где $m^{\prime}$ - масса трёх звеньев ножа, кг; $V_{m^{\prime}}$ скорость ножа в точке $m^{\prime}, \mathrm{m} / \mathrm{c} ; e-$ плечо силы $S_{2}$, M.

Подставив формулы (12) и (13) в (11), получим:

$$
S_{3}=\frac{J^{\prime} \cdot \omega_{1}^{\prime}}{d}-m \cdot \omega_{1}^{\prime} \cdot e .
$$

Ротор динамически будет уравновешен, если импульс реакции шарнира ножа равен нулю $S_{3}=0$, т.е. в нашем случае для трёх звеньев ножа:

$$
\left[\frac{J^{\prime}}{d}-m^{\prime} \cdot e\right] \cdot \omega_{1}
$$

или

$$
\frac{J^{\prime}}{d}-m^{\prime} \cdot e=0 .
$$

Так как:

$$
J^{\prime}=m^{\prime} \cdot \rho^{\prime 2},
$$

где $\rho^{\prime}$ - радиус инерции трёх звеньев ножа, то уравнение (15) примет вид:

$$
\frac{m^{\prime} \cdot \rho^{\prime 2}}{d}-m^{\prime} \cdot e=0,
$$

откуда $\rho$ ' равно:

$$
\rho^{\prime}=\sqrt{d \cdot e} .
$$

Масса одного звена зависит от калибра цепи (толщины проволоки). Ранее на ротор монтировалась Цепь 2-9х38 ГОСТ 7070-75, изготовленная из проволоки 9 мм с шагом 38 мм [2]. Масса одного звена равна 0,060 кг. Масса всей цепи составляла 0,54 кг, длина 0,36 м, что соответствует девяти звеньям. На сегодняшний день ГОСТ 7070-75 уже не действует, и цепи с такими параметрами (шаг звена 38 мм) заводами-производителями не выпускаются. ГОСТ 2319-81 (СТ СЭВ 2639-80) также отменён, взамен принят действующий ГОСТ 30188-97, но цепи имеют другие геометрические параметры [3]. Шаг звена изменён с 38 мм до 27 мм. Для обеспечения экономической эффективности мы определим новые геометрические и физические параметры цепи. 
Так как захват ротора 2,5 метра, а $r+b=$ $=1,25 \mathrm{M}$, то $b=0,36 \mathrm{M}, c=1 / 2 b=0,18$ м получим: $\rho=\sqrt{b \cdot c}=\sqrt{0,36 \cdot 0,18}=\sqrt{0,065}=0,225 \mathrm{M}$.

При том же захвате ротора 2,5 метра $L r+d=1,25 \mathrm{M}, L r=1,165 \mathrm{M}$, a $d=0,085 \mathrm{M}$, $e=1 / 2 b=0,0425 \mathrm{M}$.

$$
\begin{aligned}
\rho^{\prime} & =\sqrt{d \cdot e}=\sqrt{1,165 \cdot 0,0425}= \\
& =\sqrt{0,0495}=0,225 \mathrm{M} .
\end{aligned}
$$

Найдём момент инерции ножа $J$ относительно шарнира в обоих случаях.

Для Цепи 2-9x38 ГОСТ 7070-75:

$$
J_{9 \times 38}=m_{9 \times 38} \cdot \rho_{9 \times 38}^{2},
$$

где $n_{9 \times 38}=9$ шт. - количество звеньев цепи; $m_{9 \times 38}^{3 \mathrm{~B}}=0,060 \mathrm{M} ; m_{9 \times 38}=m_{9 \times 38}^{3 \mathrm{~B}} \cdot n_{9 \times 38}=0,54 \mathrm{K \Gamma} ;$ $\rho_{9 \times 38}=0,255 \mathrm{M}$.

$$
\begin{gathered}
J_{9 \times 38}=m_{9 \times 38} \cdot \rho_{9 \times 38}^{2}= \\
=0,540 \cdot 0,255^{2}=0,035 \mathrm{K \Gamma} \cdot \mathrm{M}^{2} .
\end{gathered}
$$

Для Цепи 9х27 ГОСТ 30188-97:

$$
J_{9 \times 27}=m_{9 \times 27} \cdot \rho_{9 \times 27}^{2},
$$

где $n_{9 \times 27}=11$ шт. - количество звеньев цепи; $m_{9 \times 27}^{3 \mathrm{~B}}=0,048 \mathrm{K \Gamma} ; m_{9 \times 27}=m_{9 \times 27}^{3 \mathrm{~B}} \cdot n_{9 \times 27}=0,53 \mathrm{K \Gamma}$; $\rho_{9 \times 27}=0,255 \mathrm{M}$.

$$
\begin{gathered}
J_{9 \times 27}=m_{9 \times 27} \cdot \rho_{9 \times 27}^{2}= \\
=0,53 \cdot 0,255^{2}=0,027 \mathrm{K \Gamma} \cdot \mathrm{M}^{2} .
\end{gathered}
$$

Сравним величины импульса при замене цепи 9х38 на 9х27 (рис. 1).

$$
S_{c}=m \cdot V_{c}=m \cdot \omega_{1} \cdot c,
$$

где $m$ - масса ножа, кг; $V_{c}$ - скорость ножа в точке $C, \mathrm{~m} / \mathrm{c} ; c$ - плечо силы $S_{c}$, м.

Частота вращения ротора $n$ составляет 2100 об/мин. или 219,9 рад/сек.

Для цепи 9х38:

$$
\begin{aligned}
& S_{c 9 \times 38}=m_{9 \times 38} \cdot V_{c 9 \times 38}= \\
& =m_{9 \times 38} \cdot \omega_{1} \cdot c_{9 \times 38} .
\end{aligned}
$$

Подставим значения и получим:

$S_{c 9 \times 38}=0,54 \cdot 219,9 \cdot 0,18=20,98 \mathrm{K \Gamma} \cdot \mathrm{M} /$ сек.

Скорость резания $S_{c 9 \times 38}=39,6 \mathrm{M} /$ сек.

Для цепи 9х27, длина 11 звеньев равна $0,315 \mathrm{M}$, поэтому $c_{9 \times 27}=0,158 \mathrm{M}$ :

$$
\begin{aligned}
& S_{c 9 \times 27}=m_{9 \times 27} \cdot V_{c 9 \times 27}= \\
= & m_{9 \times 27} \cdot \omega_{1} \cdot c_{9 \times 27} \mathrm{K \Gamma} \cdot \mathrm{M} / \mathrm{ceK} .
\end{aligned}
$$

Подставим значения и получим:

$$
\begin{aligned}
& S_{c 9 \times 27}=0,53 \cdot 219,9 \cdot 0,158= \\
& \quad=18,41 \mathrm{K \Gamma} \cdot \mathrm{M} / \text { сек. }
\end{aligned}
$$

Скорость резания $V_{c 9 \times 27}=34,7 \mathrm{M} /$ сек.

При минимальной скорости резания $V_{\min }=30,0$ м/сек процесс бесподпорного резания не будет нарушен. Замена цепи с новыми геометрическими параметрами обеспечит работоспособность ротора.

Заключение. Ранее устанавливаемая Цепь 2-9х38 ГОСТ 7070-75, изготовленная из проволоки 9 мм с шагом 38 мм, заводами-производителями не выпускаются. Принят новый действующий ГОСТ 30188-97, но цепи имеют другие геометрические параметры. Шаг звена изменён с 38 мм до 27 мм. Для обеспечения экономической эффективности были определены новые физические параметры цепи, что позволило сохранить работоспособность ротора в целом. Цепь 2-9х38 ГОСТ 7070-75 с количеством звеньев 9 шт. была заменена на Цепь 9х27-Т(8)-11 ГОСТ 30188-97 с количеством звеньев 11 шт. и разрушающей нагрузкой не менее 102,0 кН [3]. Экономическое обоснование позволило использовать новый цепной нож без изменения конструкции косилки КР-2,5К.

\section{Литература}

1. Босой О. В., Верняев О.В. и др. Теория, конструкция и расчет сельскохозяйственных машин: Учебник для вузов сельскохозяйственного машиностроения. 2-е изд. - М.: Машиностроение, 1977. - С. 269.

2. ГОСТ 7070-75 Цепи круглозвенные сварные общего назначения. - Москва: Издво стандартов, 1985. - 10 с.

3. ГОСТ 30188-97 Цепи грузоподъёмные калиброванные высокоточные. - Москва: Изд-во стандартов, 2001. - 11 с.

4. Грищенко В. В. Определение сил, действующих на нож цепного режущего аппарата роторной косилки / В.В. Грищенко // Агропромышленные машины и оборудование: сб. науч. тр. / НГМА. - Новочеркасск, 1998. C. $21-25$.

5. Погоров Т.А. Итоги полевых исследований косилки шнековой КОС-2,5 / Т. А. Погоров // Повышение надежности и эф- 
фективности машин и орудий в орошаемом земледелии: сб. науч. тр. / ЮжНИИГиМ. Новочеркасск, 1983. - С. 82-88.

6. Погоров T.A. Результаты исследований по усовершенствованию технологии окашивания каналов мелиоративных систем / Т.А. Погоров // Пути повышения эффективности орошаемого земледелия: сб. науч. тр./ ФГНУ «РосНИИПМ». - Новочеркасск, 2008. - Вып. 40. - Ч. 2. - С. 42-48.

\section{References}

1. Bosoj O.V., Vernjaev O.V. $i d r$. Teorija, konstrukcijairaschetsel'skohozjajstvennyhmashin: Uchebnik dlja vuzov sel'skohozjajstvennogo mashinostroenija. 2-e izd [Theory, design and calculation of agricultural machines: Textbook for universities of agricultural engineering. $2 \mathrm{nd} \mathrm{ed}$.]. Moscow: Mashinostroenie, 1977. — P. 269.

2. GOST 7070-75 Cepi kruglozvennye svarnye obshhego naznachenija [General purpose round-link welded Chains]. - Moscow: Izd-vo standartov, 1985. - $10 \mathrm{p}$.

3. GOST 30188-97 Cepi gruzopodjomnye kalibrovannye vysokotochnye [Load-lifting chains, calibrated, high-strength]. - Moscow: Izd-vo standartov, 2001. - $11 \mathrm{p}$.

4. Grishhenko V.V. Opredelenie sil, dejstvujushhih na nozh cepnogo rezhushhego apparata rotornoj kosilki [Determination of forces acting on the knife of the chain cutting device of the rotary mower] / V.V. Grishhenko// Agropromyshlennye mashiny i oborudovanie: sb. nauch. tr. [Agro-Industrial machines and equipment: collection of scientific works]. / NGMA. Novocherkassk, 1998. - Pp. 21-25.

5. Pogorov T.A. Itogi polevyh issledovaniı̌ kosilki shnekovoĭ KOS-2,5 [Results of field research of the screw mower KOS-2,5] / T.A. Pogorov // Povyshenie nadezhnosti i jeffektivnosti mashin $\mathrm{i}$ orudiı̌ $\mathrm{v}$ oroshaemom zemledelii: sb. nauch. tr. [Improving the reliability and efficiency of machinery and implements in irrigated agriculture: collection of scientific works] / JuzhNIIGiM. - Novocherkassk, 1983. - Pp. 82-88.

6. Pogorov T.A. Rezul'taty issledovaniǐ po usovershenstvovaniju tehnologii okashivanija kanalov meliorativnyh sistem [Results of research on improving the technology of mowing channels of reclamation systems] / T.A. Pogorov// Puti povyshenija jeffektivnosti oroshaemogo zemledelija: sb. nauch. tr. [Ways of increase of efficiency of irrigated agriculture: collection of scientific works] / FGNU «RosNIIPM». - Novocherkassk, 2008. Issue 40. — Part 2. - Pp. 42-48. 


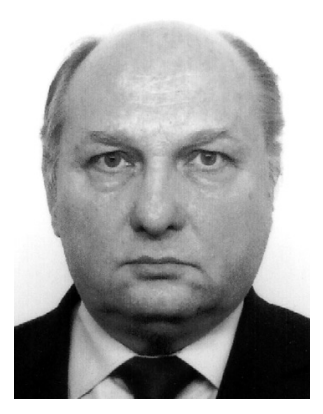

Грищенко Владимир Викторович - кандидат технических наук, доцент кафедры «Машины природообустройства» Новочеркасского инженерно-мелиоративного института имени А.К. Кортунова (филиала) Донского государственного аграрного университета. Специалист в области исследования режущих аппаратов мелиоративных и сельскохозяйственных машин и оборудования.

Grishchenko Vladimir Victorovich - Candidate of Engineering Sciences, Associate Professor of the Department «Machine Engineering», Novocherkassk Reclamation Engineering Institute named after A.K. Kortunov (branch), Donskoy State Agrarian University. Specialist in the field of research of cutting devices for reclamation and agricultural machinery and equipment.

346411, г. Новочеркасск, пер. Гайдара, 13, кв. 4

13 Gaidar ln., app. 4, 346411, Novocherkassk, Russia

E-mail: v_grishchenko@mail.ru 